\title{
Miradas CRUZadas SOBRE EL ENFOQUE TERRITORIAL DE LA RESPONSABILIDAD SOCIAL, LA GOBERNANZA Y LA SOSTENIBILIDAD
}

\author{
Guillen Calvo* \\ Oscar Iván PÉrez Hoyos** \\ María Claudia Romero Amaya*** \\ Editores invitados
}

Durante los últimos diez años, las exigencias del desarrollo territorial y local, y los retos que impone la agenda global de desarrollo implican al sector privado -las empresas y sus fundaciones- en asuntos que no han sido de su tradicional resorte. Esta situación supone repensar el papel y la contribución de esos actores a los procesos de desarrollo y nos enfrenta a ciertos cuestionamientos: ¿cómo entender la empresa en tanto actor social y no solamente económico? ¿Cuáles son las responsabilidades que tiene el sector privado en la promoción del desarrollo? ¿Dónde acaban sus responsabilidades y empiezan las de los actores públicos? ¿Cómo los distintos actores públicos, privados y del tercer sector pueden dialogar y articularse para incidir en la planeación y ejecución conjunta de proyectos en el territorio? ¿Cuáles son las características de los contextos en los que las empresas desarrollan sus operaciones y qué expectativas se generan en los otros actores ante su presencia? ¿Cómo se vincula el sector

* Agroeconomista y geógrafo de formación. Director general para América Latina de Insuco, empresa consultora especializada en la gestión social y sostenibilidad de proyectos. Docente y director del seminario "Enfoque territorial de la Responsabilidad Social Empresarial”, en la Maestría en Responsabilidad Social y Sostenibilidad de la Universidad Externado de Colombia, Bogotá (Colombia). [guilllen.calvo@insuco.com]; [https://orcid.org/00000003-3032-8544].

** Economista y magíster en Ciencias Económicas, Universidad Nacional de Colombia. Docente-investigador de la Maestría en Responsabilidad Social y Sostenibilidad de la Universidad Externado de Colombia, Bogotá (Colombia). [oscar.perez@uexternado.edu.co]; [https://orcid.org/0000-0001-8889-7446].

*** Candidata a doctora en Estudios Políticos; magíster en Gobierno y Políticas Públicas, Universidad Externado de Colombia. Docente-investigadora del Centro de Investigaciones y Proyectos Especiales (cipe), de la Facultad de Finanzas, Gobierno y Relaciones Internacionales, Universidad Externado de Colombia, en temas de Sostenibilidad, Responsabilidad Social Empresarial e Inversión Social, Bogotá (Colombia). [maria.romeroa@uexternado.edu.co]; [https://orcid.org/0000-0002-2326-5066].

DOI: https://doi.org/10.18601/16578651.n26.02 
privado con las agendas públicas y con los instrumentos de planificación socioeconómica y territorial? ¿Qué efectos e impactos tiene la inversión social que empresas y fundaciones despliegan en la transformación social, productiva e institucional de los territorios? Todos estos cuestionamientos son temas que atraviesan y son debatidos en espacios académicos, gubernamentales, del sector privado y en el seno de iniciativas multiactor.

Dos situaciones particulares convocan hoy a la reflexión. Una, nos sitúa en lo local y tiene que ver con la territorialización del desarrollo; la otra, nace de la adopción de la Agenda 2030 y nos sitúa en lo global, bajo el entendido, sin embargo, de que la consecución de metas globales depende de esfuerzos localizados y de la articulación de innovaciones a diferentes niveles de escala que sumarán en el cumplimiento de propósitos universales.

La Universidad Externado de Colombia, desde el Centro de Investigaciones y Proyectos Especiales (CIPE), particularmente desde la línea de Desarrollo Territorial Sostenible, y en el marco de su maestría en Responsabilidad Social y Sostenibilidad, viene trabajando en procesos de investigación que buscan contribuir a la reflexión y a la generación de conocimiento sobre estos asuntos.

El número de la revista Opera que hoy se presenta recoge ocho trabajos realizados por profesores, estudiantes e investigadores externos, que proponen miradas desde distintas aristas, en una conjunción de esfuerzos por diagnosticar, proponer marcos de análisis y explorar rutas de acción. Si bien el espectro de los temas abordados en este dossier es amplio, así como lo es la diversidad de contextos territoria- les analizados, un elemento común y tres temas de discusión se presentan de manera transversal a lo largo del número. El elemento común es el sector privado, los ejes de discusión son el enfoque territorial de la responsabilidad social empresarial, la gobernanza y la sostenibilidad.

Con base en estudios, mayoritariamente empíricos, se hace una revisión de casos que en Colombia y otras partes del mundo arrojan luces sobre esas nuevas formas de pensar y construir la relación entre sector privado, sostenibilidad y desarrollo territorial.

\section{HACIA UN ENFOQUE TERRITORIAL DE LA RESPONSABILIDAD SOCIAL EMPRESARIAL}

Para entender esas relaciones es necesario poner en perspectiva el cambio de mirada que la sociedad ha venido proyectando sobre el sector privado, así como la evolución en la forma de observar el territorio como unidad de análisis. En el primer caso, la contribución del sector privado partió de una percepción de agente económico cuyo único objetivo es generar beneficios económicos, con un retorno hacia el territorio en términos de generación de empleo, compra de bienes y servicios, y pago de impuestos.

Bajo esta percepción, netamente empresarial, el territorio ha sido visto como un soporte para las actividades económicas; un espacio geográfico en donde la empresa desarrolla su actividad económica vinculándose a él a partir de la necesidad de aprovechar sus recursos naturales en función de su sector de actividad. Bajo ese supuesto, la relación entre territorio y empresa se ha caracterizado por su 
naturaleza meramente transaccional, sin que se conciba un rol de la misma en la gobernanza del territorio o en las redes de colaboración que lo atraviesan.

Como se ha anunciado, en la última década, dicha relación entre el sector privado y el territorio se viene modificando. Mientras la forma de pensar el papel de la empresa en la sociedad y sus responsabilidades con ella se transforma, el territorio deja de entenderse como un espacio físico "objetivamente existente" -o como un espacio simplemente delimitado por consideraciones administrativas- para comprenderse ahora como una construcción social. Esto es, un conjunto de relaciones sociales que rigen y, a la vez, expresan una identidad y un sentido de propósitos compartidos por múltiples agentes públicos y privados.

Esa transición de una visión de "territorio soporte" a la de territorio como "recurso compartido" cambia radicalmente la forma de entender la relación entre empresa y territorio y abre la puerta a un enfoque territorial de la responsabilidad social empresarial (RSE). De esta forma, se considera al territorio como un producto social e histórico, al estar la sociedad ligada al espacio físico en que se encuentra, y constituirlo mediante un proceso de apropiación en el que evolucionan simultáneamente sistemas económicos, sociales, institucionales, culturales, políticos y ambientales (Sepúlveda, Rodríguez, Echeverri y Portilla, 2003).

Según Schetjman y Berdegué (2004), el desarrollo territorial se entiende como un proceso de transformación social, productiva e institucional en un espacio determinado que tiene el propósito de articular competitiva y sustentablemente la economía del territorio a mercados dinámicos.

Algunos de los criterios planteados por Schejtman y Berdegué (2004) para diferenciar estrategias o programas con enfoque territorial de desarrollo de otras estrategias con perspectivas más sectoriales, permiten proyectar una visión más integrada de la relación entre sector privado y territorio:

- La transformación productiva y el desarroIlo institucional se deben abordar de forma simultánea. Se debe buscar que exista una interdependencia entre la transformación productiva en territorios rurales y las diferentes políticas institucionales que apoyen el éxito de este proceso, como alianzas, redes de cooperación y espacios de concertación, entre otros. Sin estas, las diferentes propuestas no tendrían la fuerza para llevarse a cabo.

- El territorio es un espacio con identidady con un proyecto de desarrollo concertado socialmente. El territorio no debe ser entendido desde una visión operacional, sino que debe reconocerse como un espacio con identidad y múltiples interacciones en su interior. Los autores afirman que los territorios son un "proyecto de desarrollo concertado socialmente".

- Los programas de desarrollo rural con enfoque territorial deben convocar a la diversidad de agentes del territorio. Se debe promover la concertación social a través de la participación de diferentes agentes, teniendo en cuenta la heterogeneidad del territorio. 
El enfoque territorial planteado con algunas de esas características permite abordar una transición conceptual en la que las empresas, desde una visión empresa-céntrica, pasen de ver el territorio como su "área de influencia" a pensarlo como un actor clave de las dinámicas socioeconómicas de este; un actor que comparte con otros actores y que desarrolla su actividad en dicho territorio, sobre el cual genera impactos positivos o negativos.

Así pues, el enfoque territorial aplicado a la RSE, o responsabilidad territorial empresarial (Calvo y Carvallo, 2015), propone un cambio desde un enfoque transaccional -entendido como el relacionamiento unidireccional entre la empresa y las comunidades, donde el aporte del primero se fundamenta en controlar riesgos asociados al entorno y la satisfacción de expectativas de los actores sociales- a un enfoque transformacional (Bowen, Newenham-Kahindi y Herremans, 2010), en el que la empresa, las comunidades y el conjunto de actores comparten un mismo espacio y de forma corresponsable potencian una transformación social, productiva e institucional del mismo.

El enfoque territorial aplicado a la RSE permite superar la visión sectorial, ya que reconoce la complejidad y diversidad de los territorios y de sus estructuras productivas complementarias a través del reconocimiento del capital natural, humano, social, físico y financiero como soportes básicos de la economía de los territorios, con lo cual se favorece una visión multidimensional del manejo de los recursos naturales, en que lo medioambiental, lo económico, lo social y lo político-institucional interactúan en el territorio. Este enfoque abre espacios para la articulación y distribución de responsabilidades, según las competencias de cada actor en el territorio.

Así, por ejemplo, el artículo de Calvo y Carvallo plantea una reflexión sobre los mecanismos de diálogo y acción colectiva en el marco de relaciones horizontales entre Estado central, Estado local, empresas y actores de la sociedad civil, no solo para gestionar y transformar conflictos socioambientales en el contexto de proyectos de alto impacto, sino también para potenciar escenarios de corresponsabilidad entre actores, de cara al desarrollo de los territorios. El artículo nos hace reflexionar sobre la importancia de entender la intencionalidad que estructura y orienta los procesos de diálogo y cómo esa intencionalidad viene a influenciar los mecanismos de gobernanza entre actores de un territorio. Una intención estructuralmente cooperativa, incluso durante episodios de conflicto, evita el incremento de este y mantiene una dinámica de acción colectiva. Lo contrario, esto es, una intención orientada a disminuir o evitar el conflicto, conlleva una dinámica de negociación y genera respuestas transaccionales ante los intereses de ciertos actores en el territorio. Para mantenerse en el tiempo e incidir positivamente en la gobernanza del territorio esos procesos deben seguir respondiendo a las expectativas de los actores territoriales y, consecuentemente, alinearse con sus intenciones a fin de generar incentivos para su participación efectiva.

De forma complementaria, el artículo de Rey y Mazalto, basándose en un análisis de proyectos mineros desarrollados en África del Oeste en la última década, reexamina los roles y las responsabilidades de los sectores público y privado para la construcción de sinergias que 
incidan en la construcción de políticas públicas y en el desarrollo de territorios con economías extractivas. Este análisis plantea un paralelo interesante entre formas de gobernanza y "modalidades de regulación pragmáticas" que esos autores han venido desarrollando para potenciar sinergias o resolver sus diferencias.

Lo anterior nos lleva a reflexionar sobre las maneras de analizar la gobernanza a diferentes escalas.

\section{A PROPÓSITO DE LA GOBERNANZA}

La gobernanza es el producto de una realidad nueva (Kohler-Koch, 1999), en la que distintos actores participan en la toma de decisiones y la solución de problemas colectivos (Mayntz, 2002, p. 2). La comprensión de esta nueva dinámica no ha sido sencilla y se ha abordado desde distintas disciplinas.

En la esfera de las relaciones internacionales se habla de la "gobernanza global" en referencia a la vinculación de actores globales de distinta naturaleza: organizaciones internacionales, gubernamentales y no gubernamentales, que interactúan en un sistema en donde, sin contar con "un gobierno mundial constituido y revestido de una única autoridad" (Pizarro y Moraes, 2018, p. 108), se ocupan de la solución de problemas que afectan a un conjunto de naciones (Mayntz, 2002), a partir de redes de cooperación y procesos de negociación.

En los campos relativos a la gestión pública y a las políticas públicas existe un número importante de aproximaciones; pero, como afirma Rhodes (2005), se observan acuerdos al menos sobre puntos como la necesidad de cooperación y de conformación de redes para trabajar asuntos de interés público, y la difuminación de las fronteras entre los sectores público, privado y voluntario. En definitiva, es de aceptación general la existencia de un número plural de actores que entran en relación para atender asuntos tradicionalmente reservados a la esfera pública (Romero-Amaya, 2009), en donde se producen formas de gobierno más horizontales y menos jerárquicas (Cerillo, 2005; Mayntz, 1998).

La gobernanza ha sido también abordada, con menor énfasis, desde los campos de estudios del desarrollo. Querol (2002) utiliza el concepto de gobernanza para el desarrollo sostenible "para referirse al proceso y a la estructura de actores y reglas formales e informales que han de permitir alcanzar el desarrollo sostenible" (p. 1). Moreno (2013), por su parte, propone un vínculo estrecho entre la gobernanza y el desarrollo sostenible, pero entendido este, no solo desde una perspectiva ambiental, sino como aquel que promueve equilibrios con el crecimiento económico y la equidad social, además de contemplar arreglos institucionales entre distintos niveles locales, nacionales e internacionales. Afirma el autor que este es el reto fundamental que introduce la agenda 2030.

De manera general, la gobernanza, vista desde el desarrollo, hace alusión a la interacción entre un entramado de actores ubicados en espacios geográficos de distintos niveles que toman decisiones en función de la sostenibilidad.

Finalmente, los estudios de gobernanza se han ubicado también en la escala territorial. De acuerdo con Farinós (2008), una buena 
gobernanza en lo territorial tendría que considerar una serie de dimensiones y de principios aplicables a cada una de las dimensiones: 1) en la dimensión vertical multinivel -se entiende que se refiere a la relación entre los niveles central, regional y local-, los principios de responsabilidad, coordinación y cooperación; 2) en la dimensión horizontal, la relación entre territorios, el principio de cooperación; 3) también en una dimensión horizontal, pero esta vez en la relación entre sectores, el principio de coherencia; $y, 4)$ en una dimensión de participación, en la que se vinculan grupos de interés y ciudadanos a título individual, los principios de información y apertura. "El funcionamiento sistémico del conjunto de todas estas dimensiones y principios contribuirá a la meta final del desarrollo territorial sostenible" (Farinós, 2008, p. 13).

Los artículos que hacen parte de este número reflexionan sobre gobernanza a partir de los postulados de este concepto en la gestión pública, el desarrollo y la gobernanza territorial, haciendo énfasis en elementos centrales como son los procesos de participación multiactor, la coordinación entre sector público y privado, y la generación de alianzas, en tres perspectivas: como aspecto central en la modelación del desarrollo territorial; como variable de análisis del desarrollo territorial y como dinámica estructurante de procesos que aporten a la agenda global de desarrollo plasmada en los objetivos de desarrollo sostenible (oDs).

Desde la primera perspectiva, como aspecto central de modelación del desarrollo territorial, tanto Calvo y Carvallo como Leyva y Díaz plantean ejercicios de gobernanza y de acción colectiva pensados desde el ámbito territorial. Calvo y Carvallo proponen el ejercicio de la acción colectiva como una forma de tramitar los conflictos socioambientales que surgen en los territorios por la presencia de la actividad empresarial, partiendo de la distribución de responsabilidades entre los actores presentes en el territorio. En el caso del ramal ferroviario en la región del Maule, esta noción territorial se aproxima a la de paisaje cultural que permite abordar $y$ canalizar las prioridades de los distintos actores y, particularmente, de los diversos servicios sectoriales del Estado. Asimismo, exploran la necesidad de buscar nuevos modelos de gobernanza que integren la variable cultural y las estrategias de valorización de identidades culturales dinámicas en procesos de desarrollo territorial.

Desde diferentes perspectivas y contextos territoriales, los artículos de Calvo y Carvallo, Leyva y Díaz, y el de Rey y Mazalto evidencian factores que contribuyen al mantenimiento en el tiempo de un proceso de gobernanza, donde la mayoría están relacionados -e incluso enfocados específicamente- con los incentivos y las intenciones que, a su vez, están relacionados con las expectativas de los diferentes actores involucrados. Es decir, hablan de la gobernanza como un aspecto central de la modelación del desarrollo territorial.

Como variable de análisis del desarrollo territorial y con distintos énfasis, la gobernanza es utilizada por Julio et al., Romero y Pérez, Cubillos y Munévar para revisar algunos de los aspectos clave de la gobernanza: coordinación entre la actividad empresarial e instrumentos de planificación socioeconómica y del territorio; existencia de espacios de participación multiactor para la toma de decisiones sobre 
la planificación de las inversiones sociales provenientes de los privados; realización de alianzas entre los distintos actores nacionales y locales para desplegar inversiones sociales en los territorios.

En particular, Julio et al., al estudiar la presencia de la actividad minera del carbón en los instrumentos que guían el ordenamiento territorial y la planificación socioeconómica a nivel municipal en regiones productoras de este mineral en Colombia, encuentran que no es posible establecer un parámetro, pues el comportamiento por regiones, e incluso por municipios, presenta variaciones de distinta naturaleza.

Sobre este propósito, Romero y Pérez, en un artículo que busca analizar la vinculación entre la inversión social privada (ISP) y el desarrollo local a partir de 37 programas desarrollados por organizaciones (empresas y fundaciones empresariales) en Colombia, encuentran una baja alineación de los programas de inversión social ( $27 \%$ de los programas) con los planes de desarrollo local.

Cubillos propone un ejercicio diferente, pero con un sentido similar. En esta ocasión el autor, por medio del análisis cuantitativo de una base de datos de 780 proyectos de inversión social de 72 fundaciones pertenecientes a la Asociación de Fundaciones Familiares y Empresariales (AFE-Colombia), se concentra en analizar la manera como las fundaciones se articulan en los territorios desde sus proyectos de inversión, con el Plan Nacional de Desarrollo (PND) 2014-2018 y los 17 objetivos de desarrollo sostenible (oDs - CONPES 3918) (DNP, 2018). Es decir, no revisa programas, sino líneas de acción de las fundaciones, y no trabaja sobre planes de desarrollo local, sino sobre el nacional. Él observa, distinto de Romero y Pérez, una alineación directa y explícita entre los temas trabajados por las fundaciones y el PND 2014-2018.

Por otra parte, los espacios de participación y diálogo multiactor, elemento clave de la gobernanza, son revisados por Romero y Pérez, quienes encuentran una existencia baja de este tipo de espacios. En su artículo, estos autores muestran que tan solo el 18,9\% de los programas desarrollados lo explicitan.

Finalmente, sobre la realización de alianzas, los autores Pérez y Romero, Cubillos y Munévar -quien propone un análisis de los informes de sostenibilidad de diez fundaciones de empresas de los sectores de minería e hidrocarburos en Colombia (cinco fundaciones de la Asociación de Fundaciones Petroleras [AFP] y 5 de la AFE)-, encuentran en sus estudios una presencia importante de alianzas entre organizaciones de los sectores público y privado, y del tercer sector. Incluso, algunos muestran un apalancamiento con las organizaciones de cooperación internacional, aunque en menor medida. Sin embargo, dos hallazgos debilitan el potencial de las alianzas en términos de gobernanza territorial. El primero tiene que ver con la poca evidencia de acciones corresponsables en esta configuración; el segundo, con el hecho de que las alianzas no necesariamente priorizan a los actores presentes en el territorio.

Por otro lado, la gobernanza como dinámica estructurante de procesos que aporten a la agenda global de desarrollo es estudiada principalmente en los artículos de Cubillos, Munévar y Turriago -esta última, con base en un estudio de caso sectorial, analiza los esfuer- 
zos de trece bancos comerciales en Colombia para aportar, en el marco de sus iniciativas de responsabilidad social y el acogimiento del Protocolo Verde, al cumplimiento de los retos ambientales del desarrollo sostenible-. Es importante destacar que, si bien la agenda 2030 es un producto de dinámicas globales de gobernanza, la mirada de los autores es, o bien local o bien sectorial, en relación con los temas centrales de la esta.

En los artículos de Cubillos y Munévar los hallazgos no son distintos a los ya mencionados para la gobernanza territorial. Esto es, se presenta alineación de las líneas de trabajo con los ods, no se evidencian con claridad espacios de participación para la definición de las temáticas y existe una pluralidad de alianzas con características que dejan dudas sobre dinámicas fuertes de gobernanza. El artículo de Turriago muestra un elemento innovador frente a los demás: la aplicación de un principio de coherencia sectorial -adaptando las propuestas que sobre desarrollo territorial hace Farinós (2008)-, en la medida en que se trata de la vinculación de entidades del sector bancario a una iniciativa común, el Protocolo Verde.

Desde un panorama amplio, los artículos exponen la importancia del vínculo entre las dinámicas de gobernanza, el desarrollo territorial y la consecución de modelos de sostenibilidad.

\section{ARTICULACIÓN DEL SECTOR PRIVADO CON EL DESARROLLO SOSTENIBLE Y LOS ODS}

En su origen, la sostenibilidad hacía referencia al aprovechamiento racional de los recursos naturales, en el sentido de que su explotación permanente no sobrepasara los límites que podrían afectar la capacidad de los ecosistemas para recuperarse (Rodríguez, 2018).

Hoy en día, luego de hitos históricos como la Cumbre de la Tierra, celebrada en Río de Janeiro en 1992, el Plan de Aplicación de la Cumbre Mundial sobre el Desarrollo Sostenible de Johannesburgo, en 2002, y en el proceso de transición de los objetivos de desarrollo del milenio (ODM) hacia los objetivos de desarrollo sostenible (oDs), la concepción del desarrollo sostenible ha evolucionado hacia un enfoque más holístico y menos centrado en las necesidades intergeneracionales, de manera que articula el desarrollo económico, la inclusión social y la sostenibilidad ambiental. Más exactamente, "como proyecto intelectual, el desarrollo sostenible pretende comprender las interacciones entre tres sistemas complejos: la economía mundial, la sociedad global y el medio ambiente físico de la Tierra" (Sachs, 2014, p. 19).

El desarrollo sostenible también cuenta con un enfoque normativo que establece cómo deberían funcionar los tres sistemas complejos para garantizar la sostenibilidad del planeta (Tulder, 2018). A nivel internacional, la Agenda 2030 de los ons establece una serie de acciones, metas y objetivos orientados a trasladar el desarrollo internacional actual hacia una senda real de sostenibilidad. Por tanto, los oDs son un esfuerzo mundial, colectivo y multiactor que promueve "un crecimiento económico socialmente inclusivo y ambientalmente sostenible" (Sachs, 2014, p. 20).

La mayoría de los artículos del dossier basan su trabajo empírico en un enfoque explícito 
de desarrollo sostenible, bajo dos modalidades: una, análisis general de los tres sistemas complejos (Cubillos, y Romero y Pérez), y otra, un análisis parcial de los sistemas, ya sea el social o el económico (Julio et al., y Munévar) o el ambiental (Turriago).

Cubillos encuentra que los esfuerzos de las fundaciones se concentran mayoritariamente en oDs sociales y económicos, y minoritariamente en oDs ambientales. Así, por ejemplo, de acuerdo con el autor, "se encontró que los cinco primeros oDs con más proyectos relacionados son los objetivos 10, reducción de desigualdades; 11 , ciudades y comunidades sostenibles; 1 , fin de la pobreza; 5 , igualdad de género; 4, educación de calidad, mientras que los 5 oDs con menos proyectos ejecutados son los oDs 9, industria, innovación e infraestructura; 7, energía asequible y no contaminante; 6 , agua limpia y saneamiento básico; 14 , vida submarina; 15 , vida de ecosistemas terrestres". Obsérvese que los cinco oDs con más proyectos son sociales y económicos, y que cuatro de los oDs con menos proyectos son ambientales.

En esta misma línea, Romero y Pérez encuentran que "la ISP hace aportes principalmente en dos dimensiones: la sociocultural (con un énfasis altamente marcado en lo social en desmedro de lo cultural) y en la económica (con una inclinación por el apoyo a emprendimientos productivos individuales en vez de la dinamización de los sistemas económicos locales)". Los autores encuentran una articulación relativamente baja de la ISP con la dimensión ambiental, ya que pocos programas implementan esquemas de buenas prácticas ambientales (8 de 37) y casi ninguno adelanta acciones encaminadas al manejo adecuado de residuos sólidos (2), la innovación para la reducción del consumo de recursos naturales (2) y la protección de la biodiversidad y los ecosistemas (1). Por ello, Romero y Pérez concluyen que solo un porcentaje reducido de los programas apunta a la sostenibilidad en sentido estricto, pues para hacerlo tendrían que vincularse con las tres dimensiones al mismo tiempo.

Los trabajos de Julio et al. y de Munévar exploran la articulación de los sectores minero e hidrocarburos a los oDs sociales, el primero partiendo desde el core de la actividad extractiva y la segunda desde la isp ejecutada por las fundaciones empresariales. Julio et al., al hacer el análisis de la priorización de los recursos de inversión social provenientes de regalías, encuentran que temas como la promoción social, y líneas como las de inclusión social y reconciliación, y la de salud y protección social, se encuentran en un segundo orden, situación que se hace más preocupante ante la evidencia de que en los municipios con actividad minera de carbón las necesidades básicas insatisfechas están por debajo del resto del país.

Munévar, por su parte, establece que las líneas estratégicas y los programas de ISP de las fundaciones empresariales se articulan con los cinco ods sociales, aunque con diferentes énfasis: nueve fundaciones se articulan con el oDs 1, Fin de la pobreza, y con el ods 4, Educación de calidad; seis con el ods 5, Igualdad de género; dos con el ods 2, Hambre cero; y una con el oDs 3, Salud y bienestar.

Finalmente, el análisis de Turriago encuentra que los bancos comerciales hacen aportes a los oDs ambientales por medio de acciones enmarcadas en los cuatro frentes del Protocolo Verde, a saber: evaluación de riesgos 
ambientales y sociales (los bancos se articulan con el $30 \%$ de las metas ambientales), productos y programas ambientales (29\%), ecoeficiencia operacional (24\%), y capacitación y divulgación (17\%). El trabajo de Turriago evidencia que, si bien la dimensión ambiental es la menos explorada por el sector privado en el marco de sus estrategias de ISP y RSE, en Colombia algunos sectores han hecho avances interesantes en el tema que pueden servir de referente para otros sectores y actores.

En suma, la mayoría de los artículos del dossier evidencia que el desarrollo sostenible es un paradigma útil para analizar los esfuerzos del sector privado por contribuir al desarrollo -ya sea desde el core de la actividad empresarial (Julio et al.), la inversión social privada (Cubillos, Romero y Pérez, y Munévar) o la responsabilidad social (Turriago)-, y que este sector, en el marco de sus estrategias de RSE e ISP, tiende a promover más los aspectos económicos y sociales del desarrollo sostenible que los aspectos culturales, de gobernanza y ambientales.

\section{REFLEXIONES FINALES}

A propósito del tema central de este número quedan al menos cuatro campos de investigación por explorar en futuros trabajos: el primero, se orienta a analizar cómo compatibilizar la urgencia de estrategias que permitan a las empresas operar, con los tiempos que requieren procesos de acción colectiva orientados por un enfoque territorial de la responsabilidad social.

El segundo consiste en entender cómo se están articulando las organizaciones del sector privado a los ODs. Es decir, si lo están haciendo a nivel de objetivos generales, temas prioritarios o metas específicas, y si lo están haciendo con base en criterios estratégicos -como el análisis de materialidad- o criterios filantrópicos -como la satisfacción de necesidades básicas de poblaciones vulnerables-.

El tercer campo surge de un hallazgo, resultado principalmente de los trabajos de Pérez y Romero y de Cubillos, y tiene que ver con la localización de los programas de IsP de empresas y fundaciones. Los estudios arrojan una constante: la ISP se localiza prioritariamente en las zonas de influencia de las organizaciones que la financian, situación que no debería sorprender, dada la lógica estratégica que motiva una parte importante de dichas inversiones sociales. Ahora, la presencia de dichas organizaciones, a su vez, tiende a corresponder con los territorios en donde hay mayor actividad económica. Este plantea el interrogante acerca de si la Isp puede estar contribuyendo a la ampliación de las brechas de desarrollo que existen entre los departamentos y municipios del país, y al interior de los mismos. Este es un asunto que ameritaría análisis cuantitativos de impactos.

El cuarto tiene que ver con la realización de trabajos basados en unidades de análisis más generales, de tal forma que se pase del estudio de programas e iniciativas con intereses especificos sobre algunas dimensiones del desarrollo, al análisis de la operación de las organizaciones y los sectores económicos en su conjunto. Solo así podremos saber en qué grado, por ejemplo, el sector privado está tomando la Agenda 2030 como un referente en sus ejercicios de planeación, seguimiento y evaluación, y valorar si el 
aporte neto de las organizaciones y los sectores económicos al desarrollo sostenible es positivo, negativo o nulo, a todos los niveles de la escala: global, nacional y territorial.

\section{TRABAJOS ADICIONALES EN ESTE NÚMERO}

Además de los ocho artículos que componen el dossier, este número incluye tres artículos de tema libre. El primero, de Solanas y Martínez, titulado "El papel del Estado en la definición de la política argentina de hidrocarburos: ¿Hacia cuál referencial sectorial?”. Este trabajo se plantea con el objetivo de analizar la política argentina de hidrocarburos, en particular la relativa a la explotación de petróleo, con el fin de comprender las transformaciones que ha tenido en las tres últimas décadas. El segundo, escrito por Barrios, "Participación electoral en el Magdalena Medio colombiano. Un análisis del posacuerdo con las Farc", tiene como propósito analizar la configuración política en la región del Magdalena Medio antes y después del acuerdo con las FARC, tras un ejercicio de comparación entre el comportamiento de los electores en el proceso de refrendación del acuerdo de paz con las FARC y las elecciones al Senado realizadas en 2018. Y, el tercero, de Herrera-Kit, titulado "Derechos humanos: un valor imprescindible sin solución definitiva. Un debate desde el concepto de los wicked problems", en el que la autora busca demostrar que, "aunque los DD.HH. son el mecanismo indispensable de compensación de las sociedades funcionalmente diferenciadas, también podrían satisfacer características de un wicked problem para la administración que procura realizarlos a través de sus programas".

Finalmente, se presenta la reseńa de dos libros: una del tomo I de la colección de la Universidad Externado sobre corrupción en Colombia, titulado Corrupción, politica y sociedad, editado por Juan Carlos Henao y Carolina Isaza, y otra del libro Making Policies Work: First-and Second-order Mechanisms in Policy Design, editado por Capano, Howlett, Ramesh y Virani.

\section{REFERENCIAS}

Bowen, F., Newenham-Kahindi, A. y Herremans, I. (2010). When suits meet roots: The antecedents and consequences of community engagement strategy. Journal of Business Ethics, (95), pp. 297-318.

Calvo, G. y Carvallo, P. (2015). De la responsabilidad social a la responsabilidad territorial empresarial. Tercera conferencia internacional en responsabilidad social en mineria. Recuperado de https:// ifsra.org/wp-content/uploads/2018/06/ArticuloRTE_SRmining-VF_Esp.pdf

Cerillo, A. (coord.) (2005). La gobernanza hoy: 10 textos de referencia. Madrid: Instituto Nacional de Administración Pública.

Departamento Nacional de Planeación (DNP) (2018). CONPES 3918 Estrategia para la implementación de los (oDs) en Colombia. Recuperado de https://colaboracion.dnp.gov.co/CDT/CONPES/ Econ\%с3\%в3micos/3918.pdf

Farinós, J. (2008). Gobernanza territorial para el desarrollo sustentable: estado de la cuestión y agenda. Boletín de la A.E (46), 11-32. 
Kohler-Koch, B. (1999). The evolution and transformation of European governance. En B. KohlerKoch y R. Eising (eds.). The transformation of European governance (pp. 13-34). London: Routledge.

Mayntz, R. (1998). New challenges to governance theory (vol. 50). Florence: European University Institute, The Robert Schuman Centre - Jean Monet Chair Papers.

Mayntz, R. (2002). Los Estados nacionales y la gobernanza global. Revista del CLAD - Reforma y Democracia, (24), 1-8.

Moreno, M. (2013). Una lectura prospectiva de la agenda Rio+20. La emergencia de la gobernanza para el desarrollo sostenible. Revista Xihmai, $\operatorname{VIII}(15), 57-74$.

Pizarro, J. y de Moraes, C. (2018). Gobernanza global: visiones teóricas y pluralidad de actores. Relaciones Internacionales, 27(54), 107-120.

Querol, C. (2002). Gobernanza para un desarrollo sostenible en Cataluña. Conceptos, requerimientos institucionales y elementos de análisis. Barcelona: Governance on protected áreas and institutions in the mediterranean región.

Rhodes, R. (2005). La nueva gobernanza: gobernar sin gobierno. En A. Cerrillo (coord.). La gobernanza hoy: 10 textos de referencia (pp. 99-122). Madrid: Instituto de Administración Pública.
Rodríguez, M. (2018). El desarrollo sostenible en los albores de los oDs y del Acuerdo de Paris. En M. Rodríguez y M. Vélez. Gobernanza y gerencia del desarrollo sostenible (pp. 3-36). Bogotá: Universidad de los Andes.

Romero-Amaya, M. C. (2009). Responsabilidad Social Corporativa. Un nuevo pacto. Revista Zero, (23), 88-91.

Sachs, J. (2014). La era del desarrollo sostenible. Bogotá: Planeta.

Schejtman, A. y Berdegué, J. (2004). Desarrollo territorial rural. Santiago: Centro Latinoamericano para el Desarrollo Rural (RIMSP).

Sepúlveda, S., Rodríguez, A., Echeverri, R. y Portilla, M. (2003). El enfoque territorial del desarrollo rural. San José: Instituto Interamericano de Cooperación para la Agricultura (IICA).

Tulder, R. (2018). Business \& the sustainable development goals. A framework for effective corporate involvement. Rotterdam: Rotterdam School of management, Erasmus University. Recuperado de https://www.rsm.nl/fileadmin/Images_NEw/ Positive_Change/Business_and_Sustainable_ Development_Goals_-_Positive_Change_0_ Rob_van_Tulder.pdf 